\title{
Ductular reactions in the liver regeneration process with local inflammation after physical partial hepatectomy
}

\author{
Yuji Suzuki ${ }^{1,2}$, Hirokatsu Katagiri ${ }^{1}$, Ting Wang ${ }^{2}$, Keisuke Kakisaka ${ }^{2}$, Kohei Kume ${ }^{1,3}$, Satoshi S Nishizuka ${ }^{1,3}$ and \\ Yasuhiro Takikawa
}

Partial hepatectomy models in mice have been widely used for liver regeneration studies. A typical procedure removes $\sim 2 / 3$ of the liver by lobular ligation without tissue dissection. However, hepatectomy in humans involves physical damage (ie, physical partial hepatectomy, PPHx). Therefore, the liver regeneration process after PPHx should involve reactions to acute local injury followed by systematic remodeling. To clarify the liver regeneration process after PPHx, we used a murine liver injury model that mimics the actual human surgical procedure. A 20-30\% PPHx was performed by transection of the left lobe of the liver using an ultrasonically activated scalpel in mice. Gene expression and morphological characteristics were analyzed during the liver regeneration process. Liver weight continuously increased by hypertrophic reaction of hepatocytes, whereas Ki67 staining showed hepatocyte proliferation. At the transected border, emergence of ductular reactions, a representative process of hepatic tissue remodeling that contain liver stem/progenitor cells, were observed. Gene expression of the transected border and non-damaged lobes revealed that inflammatory cytokine- and extracellular matrix-associated genes were significantly upregulated at the transected border. Our PPHx model triggered local extracellular matrix remodeling that resulted in ductular reactions. These processes occurred during the tissue repair process in local inflammatory responses as well as compensatory hepatocyte hypertrophy of the entire liver. These findings may provide insight for elucidating the mechanism of tissue repair and regeneration of the liver after PPHx. Laboratory Investigation (2016) 96, 1211-1222; doi:10.1038/labinvest.2016.97; published online 12 September 2016

Partial hepatectomy is performed on the premise that the remnant liver will regenerate by compensatory growth of the remaining segments. ${ }^{1,2}$ In rodents, a typical procedure removes $\sim 2 / 3$ of the liver by lobular ligation without tissue dissection (ie, traditional partial hepatectomy). ${ }^{3,4}$ Human liver is not divided into multiple lobes, which makes segmental hepatectomy difficult. Therefore, the hepatectomy procedure is different in rodents and humans due to the anatomical difference. In general, hepatectomy in humans involves physical damage (ie, physical partial hepatectomy, PPHx), and, therefore, the liver regeneration process after PPHx should involve reactions as a result of the acute local injury followed by systematic remodeling.

The liver has a robust regenerative capacity with different modes of regeneration according to the type and extent of injury. In the situation of the traditional partial hepatectomy model, liver mass is restored by hypertrophic reaction and replication of existing hepatocytes with or without minimal contribution of liver stem/progenitor cells (LPCs). ${ }^{2,5}$ In comparison, when the proliferation of hepatocytes is impaired as a result of acute or chronic broad hepatic injury, LPCs emerge and expand forming duct-like structures, which are known as ductular reactions. ${ }^{6,7}$ LPCs are thought to contain some population of cells that have the capacity to differentiate into both hepatocytes and biliary cells, which eventually contribute to tissue repair. ${ }^{8,9}$ Ductular reactions are seen in a wide variety of human acute and chronic liver diseases. ${ }^{10}$ Although the mechanism of the ductular reaction is not fully understood, growth factors/cytokines released from the local microenvironment resulting in changes in the extracellular matrix (ECM) have been thought to be important components that elicit the ductular reaction. ${ }^{11,12}$

In this study, we used a murine liver injury model that mimics the actual human surgical procedure to clarify the liver regeneration process after PPHx. Using this model, we observed prompt ductular reactions localized to the damaged

${ }^{1}$ Molecular Therapeutics Laboratory, Department of Surgery, School of Medicine, Iwate Medical University, Morioka, Japan; ${ }^{2}$ Division of Hepatology, Department of Internal Medicine, School of Medicine, Iwate Medical University, Morioka, Japan and ${ }^{3}$ Institute of Biomedical Sciences, Iwate Medical University, Yahaba, Japan

Correspondence: Dr SS Nishizuka, MD, PhD, Molecular Therapeutics Laboratory, Department of Surgery, School of Medicine, Iwate Medical University, Morioka 020-8505, Japan

E-mail: snishizu@iwate-med.ac.jp

Received 13 January 2016; revised 27 July 2016; accepted 9 August 2016 
border during the wound healing process. Moreover, we found that the remodeling of ECM with an acute inflammation was a key molecular process associated with the emergence of ductular reactions. These findings provide insight for elucidating the mechanisms of tissue repair and regeneration after $\mathrm{PPHx}$.

\section{MATERIALS AND METHODS Animal Experiments}

Eight to ten-week-old female C57BL/6J and SCID C.B-17/ ICR-SCID/SCID mice (CLEA Japan, Tokyo, Japan) were used for the experiments. All animal experiments in this study were approved by Iwate Medical University Ethical Committee for Animal Experiment Regulation (25-038). Mice were anesthetized by inhalation of isoflurane $(2.5 \% \mathrm{v} / \mathrm{v})$. After a $2 \mathrm{~cm}$ laparotomy, PPHx was performed by transection of the left lobe of the liver using a harmonic scalpel (Harmonic Focus, Ethicon Endo-Surgery, Blue Ash, OH, USA), which is an ultrasonically activated surgical device for tissue dissection without lobular ligation, to remove $\sim 20-30 \%$ of the entire liver. The average length of the liver transection line was $\sim 10 \mathrm{~mm}$. Regenerating liver samples were harvested at 24, 48, and $72 \mathrm{~h}$ after the PPHx, and then formalin fixed or embedded in OCT compound (Sakura Finetek, Tokyo, Japan) for further analysis. Mice that underwent only laparotomy (sham operation) were used as a control. Sham-operated livers were harvested $24 \mathrm{~h}$ after laparotomy for further analysis.

\section{Histological Evaluation}

Paraformaldehyde-fixed paraffin-embedded (FFPE) (4\%) liver tissue blocks were cut into $3 \mu \mathrm{m}$ sections and stained with hematoxylin and eosin.

\section{Apoptotic Cell Detection}

Apoptosis was evaluated by terminal deoxynucleotidyl transferase-mediated deoxyuridine triphosphate nick-end labeling (TUNEL) assay using the In Situ Cell Death Detection Kit, POD (Roche diagnostics, Mannheim, Germany). TUNEL staining was performed on the transected and remnant medial lobes at each time point after the PPHx.

\section{Immunohistochemistry}

The following primary antibodies were used for colorimetric immunohistochemical staining on paraffin-embedded liver sections: rabbit anti-Ki67 antibody (1:300; RM-9106, Thermo Scientific, Fremont, CA, USA); mouse anti- $\alpha$-smooth muscle actin ( $\alpha$-SMA) antibody (1:600; MS-113-P, Thermo Scientific); rabbit anti-matrix metalloproteinase (MMP)-9 antibody (1:75; ab124513, abcam, Cambridge, UK); and rabbit anti-cytokeratin 19 (CK19) antibody (1:50; ab15463, abcam). After primary antibody incubation, the FFPE sections were incubated with peroxidase-labeled anti-mouse or anti-rabbit secondary antibodies (Histofine Simple Stain Max-PO Kit, Nichirei, Tokyo, Japan). Then, 3,3'-diaminobenzine was used for colorimetric signal development in the presence of peroxidase, which was conjugated with the secondary antibody (K3468, DAKO, Carpinteria, CA, USA). The sections were then counterstained with hematoxylin to visualize nuclei.

For immunofluorescent detection, the following primary antibodies were used on frozen sections $(6 \mu \mathrm{M})$ : rabbit antiCK19 antibody (1:40); rat anti-Thy1 antibody (1:20; 553011, BD Biosciences, San Diego, CA, USA); rat anti-epithelial cell adhesion molecule (EpCAM) antibody (1:150; 552370, BD Biosciences); rat anti-A6 antibody (1:100; gift from Valentina Factor, NIH); and rabbit anti-Ki67 antibody (1:300). All primary antibodies, except the anti-Ki67 antibody, were detected with one of the following secondary antibodies: antirabbit Alexa Fluor 488 (1:300; A21206, Life Technologies, Carlsbad, CA, USA) or anti-rat Alexa Fluor 594 (1:300; A11007, Life Technologies). The anti-Ki67 primary antibodies were incubated with peroxidase-labeled anti-rabbit secondary antibody (Histofine Simple Stain Max-PO Kit, Nichirei). Bound secondary antibody was detected by treating with tyramide-Cy3 (NEL704A001KT, Perkin Elmer Life Sciences, Waltham, MA, USA). Nuclei were counterstained with 4'-6diamidino-2-phenylindole (DAPI). The images were obtained by laser confocal microscopy (Nikon, Tokyo, Japan). To quantify the size of hepatocytes, Alexa Fluor 488 phalloidin (1:300; A12379, Life Technologies) was used to recognize the outline of hepatocytes. The average size of hepatocytes was quantified using ImageJ software (NIH Image; http://rsb.info. nih.gov/ij/).

\section{cDNA Microarray Analysis}

Total RNA was extracted using an RNeasy Mini kit (Qiagen, Tokyo, Japan) according to the manufacturer's protocol. RNA samples were extracted from $3 \mathrm{~mm}^{3}$ specimens of liver tissue from the transected border of SCID mice at each time point. The concentration and purity of the total RNA were determined using a SmartSpec Plus Spectrophotometer (Bio-Rad, Hercules, CA, USA). Gene expression profiling was performed according to the manufacturer's instructions (Agilent Whole Mouse Genome Microarray, $4 \times 44 \mathrm{~K}$, Agilent Technologies, Santa Clara, CA, USA). The microarray data were analyzed by Genespring GX12 software (Agilent Technologies). Genes with $>3.0$-fold up- or downregulation compared with sham samples were considered differentially expressed. Differentially expressed genes at any single time point after the PPHx were analyzed by one-way hierarchical clustering with a Euclidean distance measurement and a Ward linkage in the Genespring GX12 software. Genes from major clusters were further analyzed using the Database for Annotation, Visualization and Integrated Discovery (DAVID; http://david.abcc.ncifcrf.gov) for gene ontology classification.

\section{RNA Preparation and Quantitative Reverse Transcription PCR}

For this assay, C57BL/6J mice were used. Total RNA was extracted from $3 \mathrm{~mm}^{3}$ specimens of medial intact lobe and of 
the transected border of the damaged left lobe, respectively. First-strand cDNA was synthesized from $1 \mu \mathrm{g}$ of total RNA using the ReverTra Ace qPCR RT Kit (Toyobo, Osaka, Japan). The synthesized cDNA was used for real-time PCR (LightCycler: Roche diagnostics, Mannheim, Germany) with FastStart SYBR Green Master (Roche diagnostics) as a fluorophore. RNA expression was normalized to the housekeeping gene 18s rRNA. Primer sequences are listed in Supplementary Table 1.

\section{Proliferation of Mouse LPCs}

An EpCAM positive liver progenitor cell line from the liver of a 3.5-diethoxycarbonyl-1.4-dihydrocollidine-fed adult mouse was kindly provided by Professor Atsushi Miyajima and Dr Minoru Tanaka of University of Tokyo. ${ }^{13}$ Serum-starved LPCs were treated with recombinant murine tumor necrosis factor- $\alpha$ (TNF- $\alpha$ ) (rm TNF- $\alpha$ : Wako, Osaka, Japan) with or without BAY11-7082 (Wako), which irreversibly inhibits IkappaB- $\alpha(\mathrm{I} \kappa \mathrm{B}-\alpha)$ phosphorylation, resulting in the disruption of nuclear factor-kappaB $(\mathrm{NF} \kappa \mathrm{B})$ nuclear translocation and activation. ${ }^{14}$ Cells were stimulated with the reagents for $24 \mathrm{~h}$ to assess Cyclin D1 expression and for 15 min to assess $\mathrm{p}-\mathrm{I} \kappa \mathrm{B}-\alpha$ expression.

\section{Cell Viability Assay}

Serum-starved LPCs $\left(5.0 \times 10^{4}\right.$ cells $\left./ \mathrm{ml}\right)$ were treated with TNF- $\alpha$, BAY11-7082, or their combination for $24 \mathrm{~h}$. The number of viable cells was counted using a Cell Count Reagent SF (Nacalai Tesque, Tokyo, Japan). ${ }^{15}$ Light absorbance was measured at $450 \mathrm{~nm}$ with a microplate photometer (Immuno Mini NJ-2300: InterMed, Tokyo, Japan).

\section{Western Blot}

Total protein was extracted from the LPCs using a Total Protein Extraction Kit (BioChain Institute, Hayward, CA, USA). Five micrograms of protein from each sample was separated using $10 \%$ sodium dodecyl sulfate polyacrylamide gel electrophoresis and electrotransferred onto a polyvinylidene difluoride membrane. Immunobloting was performed using rabbit anti-p-I $\kappa \mathrm{B}-\alpha$ (Ser32) (1:1000; \#2859, Cell Signaling Technology, Danvers, MA, USA); mouse antiCyclin D1 (1:1000; \#2926, Cell Signaling Technology); and mouse anti- $\beta$-actin (1:500; sc-47778, Santa Cruz Biotechnology, Dallas, TX, USA). Horseradish peroxidase-conjugated sheep anti-mouse IgG (1:5000; NA9310V, GE healthcare, Little Chalfont, Buckinghamshire, UK) or donkey anti-rabbit IgG (1:5000; N9340V, GE Healthcare) were used as secondary antibodies. Immunoreactive bands were detected by chemiluminescence using the ECL Prime Western Blotting Reagent (GE Healthcare).

\section{Statistical Analysis}

Unless otherwise noted, data were expressed as the mean \pm s.e.m. Statistical analyses including Student's unpaired, two-tailed $t$-test were conducted using GraphPad
Prism, version 6 (GraphPad Software, San Diego, CA, USA). Differences were considered significant when the $P$-value was $<0.05$.

\section{RESULTS}

\section{Mouse Model of PPHx}

In contrast to a traditional partial hepatectomy that simply removes the medial and left lateral lobes by ligating the pedicle, our PPHx model employs a harmonic scalpel that removes $\sim 20-30 \%$ of the entire liver (Figure 1a). The width of the ablation band of the liver resection was $\sim 1 \mathrm{~mm}$, which corresponded to the coagulated tissue (Figure 1b). Figure 1c shows histological characterization of the wound edge tissue at specific time points after the PPHx. At $24 \mathrm{~h}$, massive hepatic parenchymal injury was observed in the damaged area. TUNEL assays exhibited a broad area of cells with DNA fragmentation in the damaged area in contrast to non-damaged areas (Figure 1c and Supplementary Figure 1). At $48 \mathrm{~h}$, substantial infiltration of neutrophils and lymphocytes was observed, while small duct-like structures were seen along the damaged border. By $72 \mathrm{~h}$, infiltrating cells further accumulated along the damaged border. The cells comprising the duct-like structures were CK19 positive, suggesting that ductular reactions had been initiated. We also confirmed that the negative control showed no staining (Supplementary Figure 2).

\section{PPHx Induces Ductular Reactions at the Damaged Border}

To investigate the process of ductular reactions that emerged at the transected border, we next performed CK19 and Thyl immunofluorescent staining. There were almost no CK19positive cells at the transected border at $24 \mathrm{~h}$ (Figure 2a). At 48 h, CK19-positive cells (ie, ductular reactions or LPCs) were observed in some areas of the transected border (Figure 2b). CK19-positive duct-like structures were also observed, especially in the periportal areas adjacent to the damaged area (Figure 2c). By $72 \mathrm{~h}$, the number of CK19positive cells had increased along the damaged border (Figure 2d). In addition, Thyl-positive cells, known as a niche for LPCs, ${ }^{16}$ also appeared in the vicinity of the ductular reactions (Figure 2e). The CK19-positive area at the damaged border was significantly increased over the time course (Figure 2f). Thyl-positive areas also appeared to be increased by $48 \mathrm{~h}$, but the difference was not statistically significant. In intact tissue areas, only preexisting structured bile ducts stained positive for CK19 and apparent ductular reactions were not observed. We further examined the expression of A6 and EpCAM to characterize newly formed bile duct-like structures (ie, ductular reactions) induced by PPHx. Ductular reactions showed A6 and EpCAM-positive cells adjacent to the transected border (Supplementary Figures $3 \mathrm{a}$ and $\mathrm{b}$ ). These findings suggest that physical liver damage by $\mathrm{PPHx}$ induces an LPC response that may form bile duct-like structures. 
a

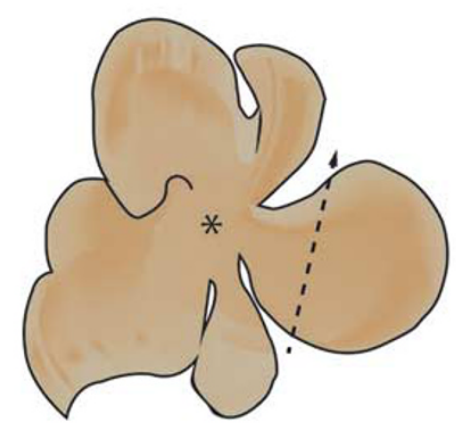

c

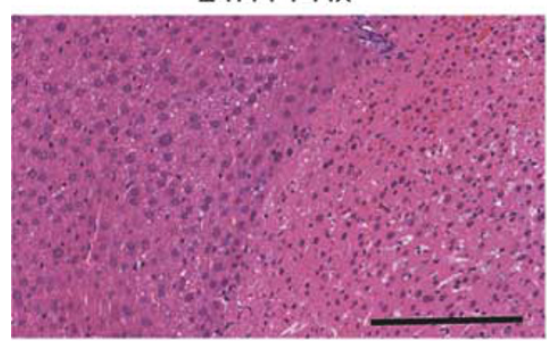

$48 \mathrm{H} \mathrm{PPHx}$

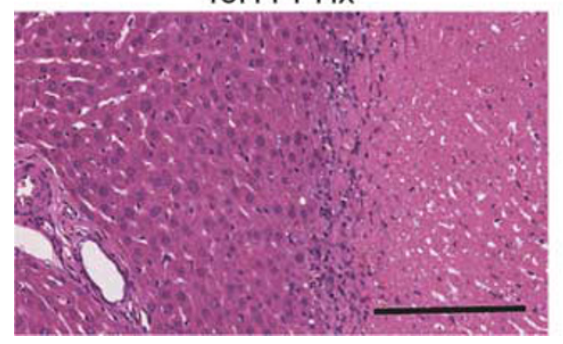

b

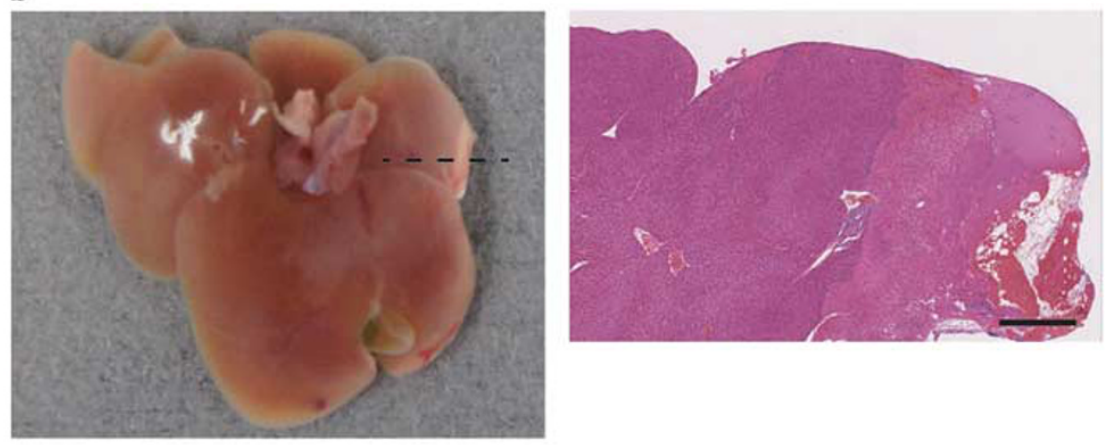

$24 \mathrm{H} \mathrm{PPHx}$ distant

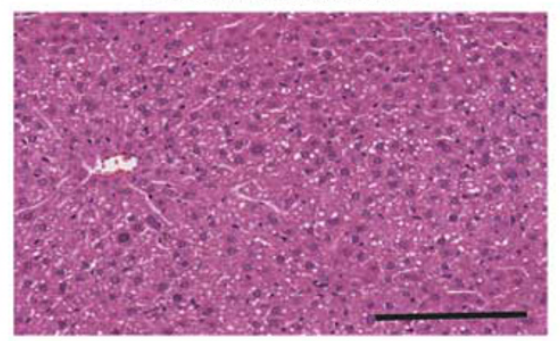

$72 \mathrm{H} \mathrm{PPHx}$

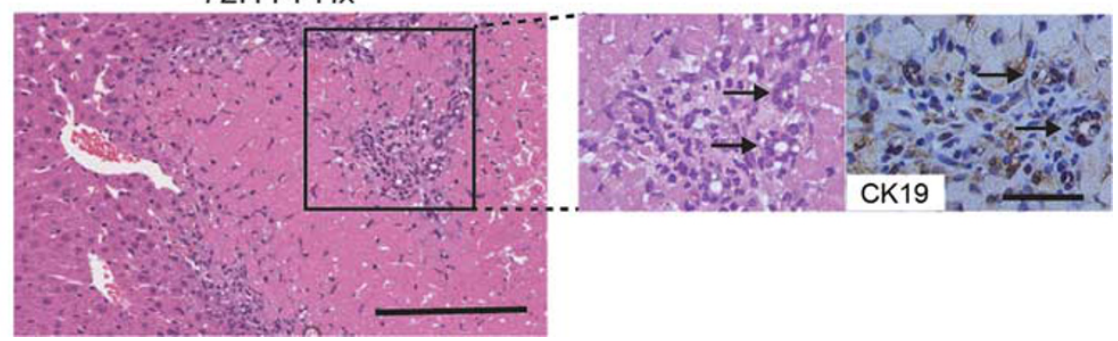

Figure 1 Schematic of the PPHx and liver section histology after the PPHx. (a) Conventional ligation point of traditional 2/3 partial hepatectomy (asterisk). The dashed line indicates the transected line in the left lobe in this study. (b) Macroscopic view and hematoxylin and eosin staining of the transected liver at $24 \mathrm{~h}$ after the PPHx. The right side of the hematoxylin and eosin staining is the coagulated area. Scale bar, $500 \mu \mathrm{m}$. (c) Histological examination of transected liver at the indicated time points. Scale bar represents $200 \mu \mathrm{m}$ except for the right lower panel. In the right lower panel, the arrows indicate that CK19-positive duct-like structures. Scale bar, $50 \mu \mathrm{m}$.

\section{PPHx Does Not Affect Hypertrophic Reaction and Proliferation of Hepatocytes in the Remnant Liver}

After traditional partial hepatectomy in mice, hypertrophy occurs throughout the entire liver followed by proliferation of hepatocytes. ${ }^{2}$ To investigate whether hypertrophy and proliferation of hepatocytes are involved in the wound healing process, we monitored microscopic and macroscopic features after PPHx in the damaged front and intact remnant liver.

The liver weight increased in a time-dependent manner (Figure 3a). The transected site was identified grossly with no indication of adjacent tissue damage (Supplementary Figure 4). Microscopically, we examined the size of hepatocytes, which play a critical role in restoring liver volume after partial hepatectomy. The size of hepatocytes increased up to $24 \mathrm{~h}$ and slightly decreased from 48 to $72 \mathrm{~h}$ (Figure $3 \mathrm{~b}$ ). We also examined the local proliferative activity of hepatocytes with Ki67 staining, but there was no significant difference between the transected left and remnant medial lobes at any time points (Figure 3c). This result was in agreement with a previous report that $50 \%$ of PPHx induced hepatocyte proliferation in the entire liver. ${ }^{17}$ Cyclin D1-encoding gene Cond1, which is an alternative cellular proliferation marker, ${ }^{18}$ was also significantly upregulated at $72 \mathrm{~h}$, but there was no significant difference between the transected and intact lobes (Figure 3d). In contrast, Ki67-positive proliferating cells were significantly increased in the ductules at the transected border, suggesting that duct-like structures located at the transected border after PPHx have increased proliferative activity (Supplementary Figures $5 \mathrm{a}$ and b).

\section{Gene Expression Profile in the Damaged Area Reveals an Inflammatory Response and Matrix Metalloproteinase \\ Activities}

We hypothesized that the wound region provides a microenvironment that mobilizes LPCs in response to injury. To determine the molecular profiles of the wound region, we 


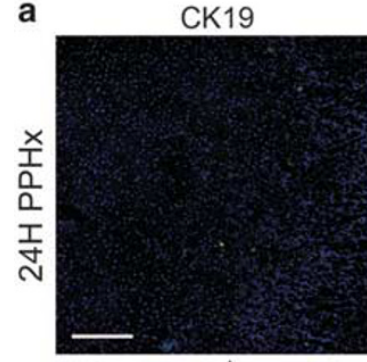

$\boldsymbol{\Delta}$

b

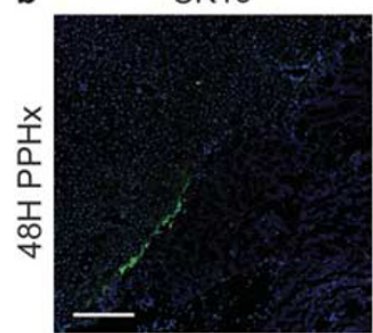

c

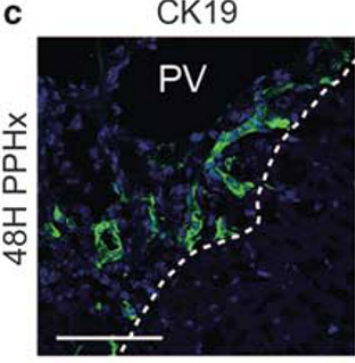

d $\quad$ CK19

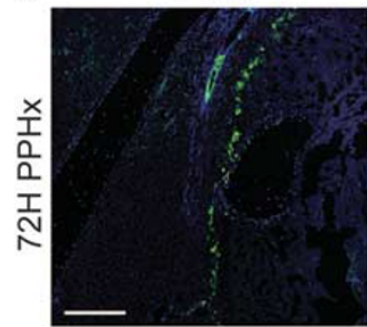

e

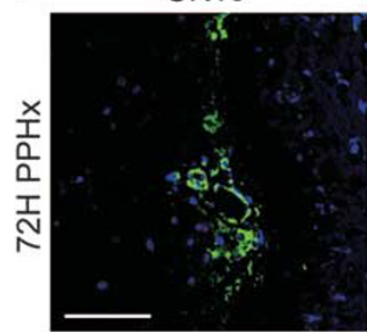

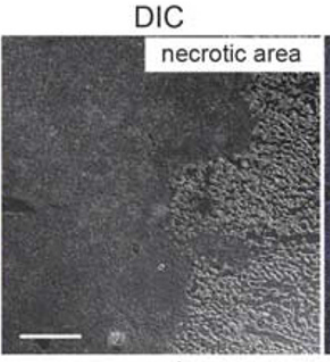

$\boldsymbol{\Delta}$

DIC

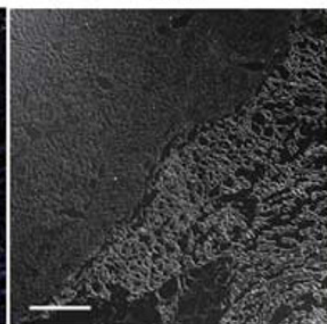

DIC

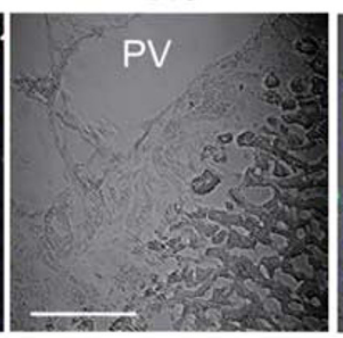

DIC

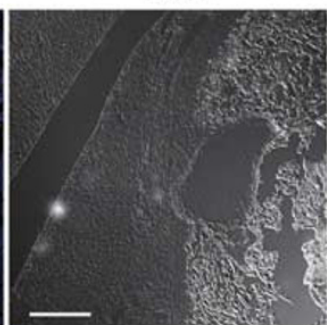

Thy1

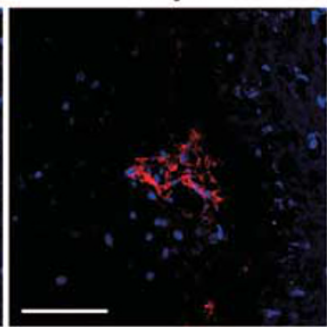

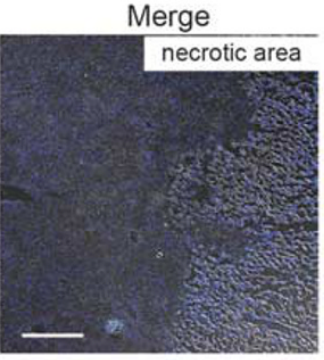

Merge

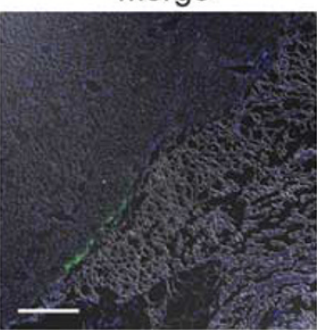

Merge

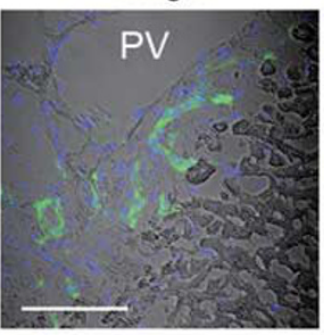

Merge

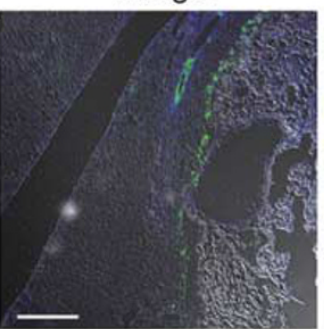

Merge

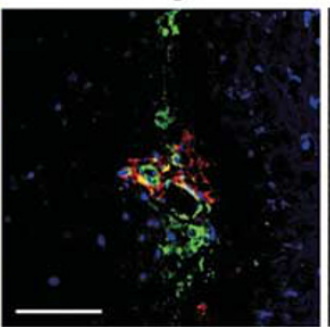

f

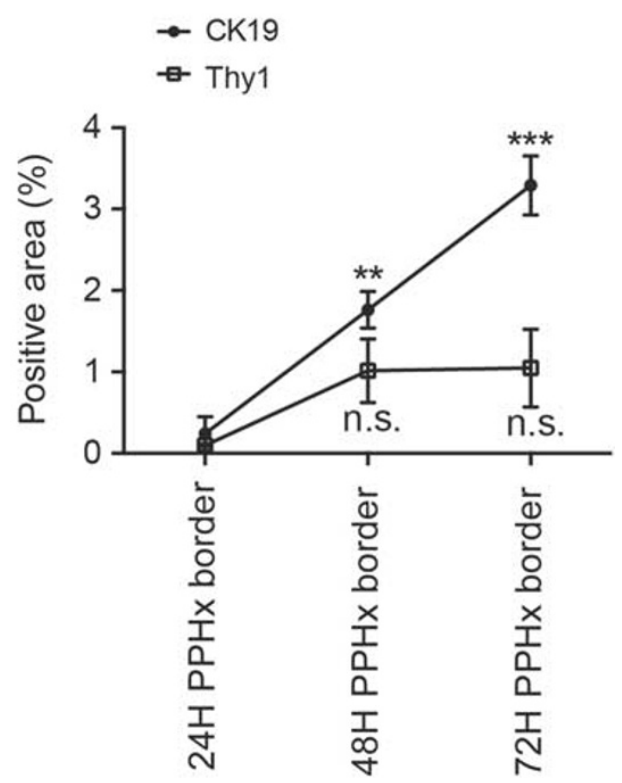

Figure 2 CK19 and Thy1 immunofluorescent staining after a PPHx. (a) CK19-immunostaining, DIC, and merged images at $24 \mathrm{~h}$ after the PPHx. Arrowheads indicate the transected line. Scale bar, $300 \mu \mathrm{m}$. (b) CK19-immunostaining, DIC, and merged images at $48 \mathrm{~h}$ after the PPHx. Scale bar, $300 \mu \mathrm{m}$. (c) CK19-positive duct-like structures in periportal areas adjacent to the damaged area. Dashed line indicates the transected border. Scale bar, $100 \mu \mathrm{m}$. (d) CK19-immunostaining, DIC, and merged images at $72 \mathrm{~h}$ after PPHx. Scale bar, $300 \mu \mathrm{m}$. (e) Double immunostaining of CK19 (green) and Thy 1 (red). Scale bar, $100 \mu \mathrm{m}$. (f) CK19- and Thy1-positive areas were quantified by immunostaining images. Student's $t$-test was performed between each time point and $24 \mathrm{~h}$ after the PPHx. Mean \pm s.e.m. $(n=3),{ }^{* *} \mathrm{P}<0.01,{ }^{* * *} \mathrm{P}<0.001$, n.s., not significant. DIC, differential interference contrast. PV, portal vein. 


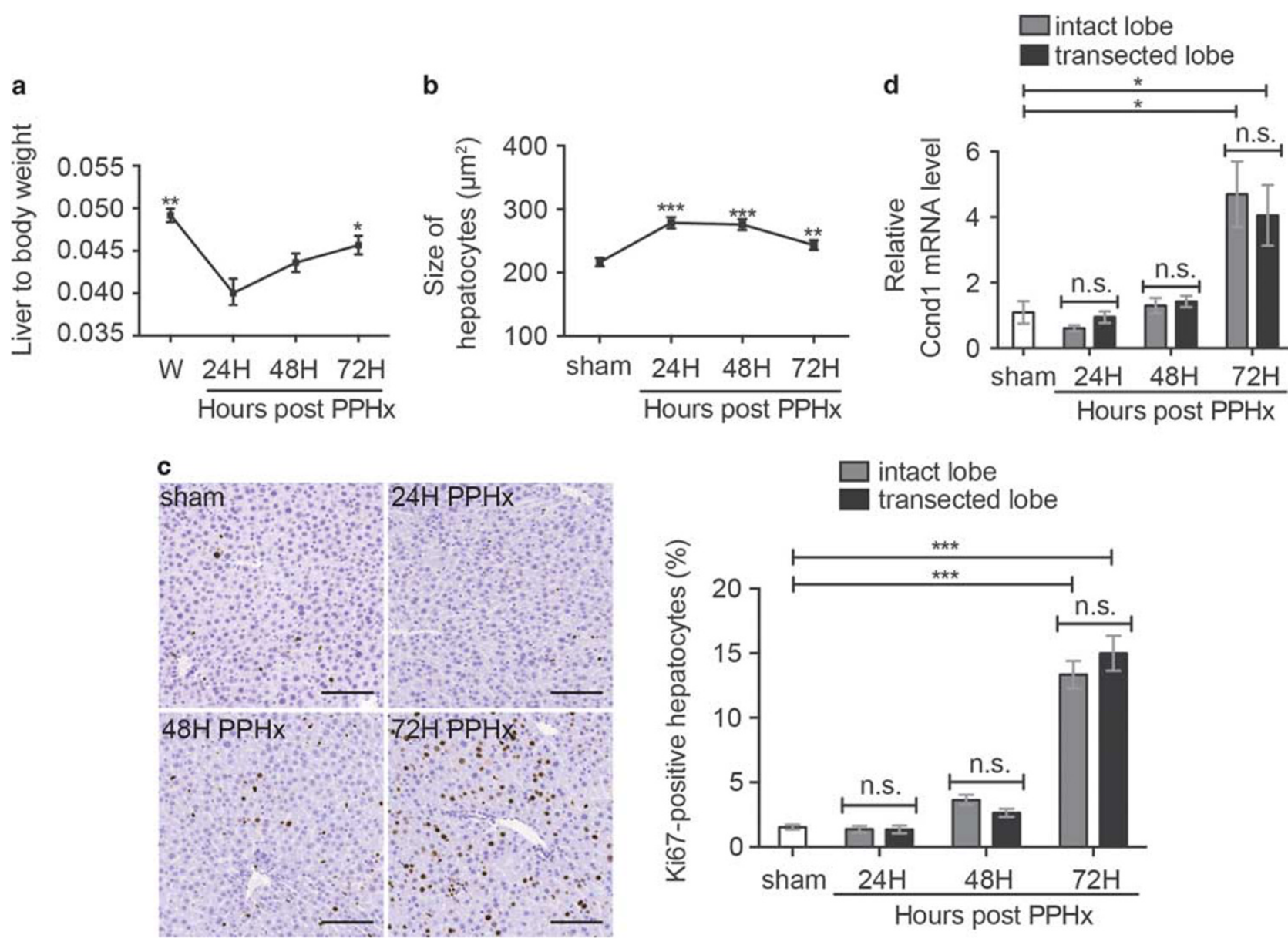

Figure 3 Hypertrophic reaction and proliferation of hepatocytes in the remnant liver after the PPHx. (a) Changes of liver to body weight ratios after the PPHx. Student's $t$-test was performed between each time point and $24 \mathrm{~h}$ after PPHx. Mean \pm s.e.m. $(n=3),{ }^{*} P<0.05$, ${ }^{* *} P<0.01$. (b) Size of hepatocytes after PPHx. Student's $t$-test was performed between sham-operated liver and each time point. Mean \pm s.e.m. $(n=3),{ }^{* *} P<0.01,{ }^{* * *} P<0.001$. (c) Ki67 staining on liver sections of transected left lobe and intact medial lobe. Scar bars, $100 \mu$ m. Mean \pm s.e.m. $(n=3),{ }^{* * *} P<0.001$. (d) Ccnd1 mRNA expression levels in transected lobe and intact lobe. Fold induction was estimated on sham-operated liver. Mean \pm s.e.m. $(n=3)$, ${ }^{*} P<0.05$.

performed microarray analysis for genes specifically expressed in the transected border. A total of 3057 differentially expressed genes were subjected to one-way hierarchical clustering and represented in a heatmap of the time course (Supplementary Figure 6). Differentially expressed genes were clustered by the time course and each cluster was related to functional annotation using DAVID. We selected up to the top four genes from ontology terms in each cluster based on the literature (Table 1). ${ }^{19-21}$ We noted that genes encoding pro-inflammatory cytokines (eg, Tnf, Il6, and Illb), CC and CXC chemokines, and their receptors (eg, $\mathrm{Cxcl} 2, \mathrm{Cxcr2}, \mathrm{Ccl} 2$, and $C \mathrm{cr} 2$ ) were upregulated transiently at $24 \mathrm{~h}$, and $32 \%$ of them returned to control levels at $48-72 \mathrm{~h}$ after the PPHx. Genes in ECM-associated genes, such as collagen transcripts (eg, Colla1, Colla2, Col3a1, Col4a1, and Col4a2), S100a4 (which promotes liver fibrosis by activating hepatic stellate cells (HSCs)), ${ }^{22}$ MMP genes (eg, Mmp2, 3, 7, 9, and 13), and tissue inhibitors of metalloproteinase genes (Timp1 and Timp2), were upregulated at $24 \mathrm{~h}$ and remained at their highest levels for up to $72 \mathrm{~h}$. From 48 to $72 \mathrm{~h}$, cell cycle-associated genes were upregulated, which is consistent with previous reports indicating that genes in both cell cycle and DNA replication categories are upregulated after a traditional partial hepatectomy. ${ }^{23,24}$ In fact, Ki67 staining showed that hepatocytes entered the cell cycle at $48 \mathrm{~h}$. However, no difference was seen in Ki67 positivity of hepatocytes between the transected and intact lobes (Figure 3c). In addition, no difference was seen in the level of $C c n d 1$ mRNA expression between the transected and intact lobes (Figure 3d). In contrast, inflammatory cytokine- and ECM-associated genes do not represent major categories for the traditional partial hepatectomy. ${ }^{25}$ We confirmed that a subset of these genes were upregulated at the transected border by quantitative reverse transcription PCR (Figures 4a and $b$ ). Taken together, these data suggest that remodeling of the ECM with an acute inflammation is a key molecular process of wound healing after PPHx. In addition, we confirmed that the expression levels of Spp1 and Afp mRNA, 
Table 1 Functional annotation clustering using DAVID

\begin{tabular}{|c|c|c|c|}
\hline Cluster & Gene ontology term & Count & $P$ \\
\hline \multirow[t]{4}{*}{ Upregulated at $24-72 \mathrm{~h}$ (181 probe sets) } & Response to wounding & 18 & $2.9 \mathrm{E}-9$ \\
\hline & Extracellular region & 40 & $3.7 \mathrm{E}-9$ \\
\hline & Extracellular region part & 26 & $1.0 \mathrm{E}-8$ \\
\hline & Chemokine activity & 8 & $2.7 \mathrm{E}-8$ \\
\hline \multirow[t]{4}{*}{ Upregulated at $24 \mathrm{~h}$ (626 probe sets) } & Inflammatory response & 15 & $2.1 \mathrm{E}-4$ \\
\hline & Defense response & 21 & $8.9 \mathrm{E}-4$ \\
\hline & Response to wounding & 17 & $2.1 \mathrm{E}-3$ \\
\hline & Acute inflammatory response & 6 & $2.5 E-2$ \\
\hline \multirow[t]{2}{*}{ Upregulated at $48 \mathrm{~h}$ (307 probe sets) } & MHC class II protein complex & 4 & $6.6 \mathrm{E}-5$ \\
\hline & Antigen processing and presentation of peptide antigen via MHC class $\|$ & 4 & $2.4 \mathrm{E}-4$ \\
\hline \multirow[t]{3}{*}{ Upregulated at $72 \mathrm{~h}$ (477 probe sets) } & Extracellular matrix & 20 & $1.3 \mathrm{E}-6$ \\
\hline & Proteinaceous extracellular matrix & 19 & $3.0 \mathrm{E}-6$ \\
\hline & Extracellular region part & 31 & $1.8 \mathrm{E}-5$ \\
\hline \multirow[t]{3}{*}{ Upregulated at 24 and $72 \mathrm{~h}$ (319 probe sets) } & Extracellular matrix & 20 & $5.3 \mathrm{E}-8$ \\
\hline & Extracellular region part & 32 & $7.1 \mathrm{E}-8$ \\
\hline & Proteinaceous extracellular matrix & 19 & $1.5 \mathrm{E}-7$ \\
\hline \multirow[t]{3}{*}{ Upregulated at $48-72 \mathrm{~h}$ (357 probe sets) } & Cell cycle & 72 & $7.5 E-46$ \\
\hline & M phase & 50 & $2.0 E-39$ \\
\hline & Cell cycle phase & 51 & $2.0 \mathrm{E}-37$ \\
\hline \multirow[t]{3}{*}{ Downregulated at any one point of time (564 probe sets) } & Extracellular region part & 28 & $1.2 \mathrm{E}-3$ \\
\hline & Extracellular matrix & 14 & $5.3 \mathrm{E}-3$ \\
\hline & Proteinaceous extracellular matrix & 13 & $9.8 \mathrm{E}-3$ \\
\hline \multirow[t]{2}{*}{ Downregulated at $24-72 \mathrm{~h}$ (226 probe sets) } & Eye development & 5 & $9.6 \mathrm{E}-3$ \\
\hline & Sensory organ development & 6 & $1.2 \mathrm{E}-2$ \\
\hline
\end{tabular}

Functional enrichment of data presented in each cluster. $P=P$-value.

which are a bile duct-associated gene and progenitor marker, respectively, were upregulated at the transected border.

Among the genes of additional interest, Krt19 mRNA, which encodes the CK19 protein, was significantly upregulated at the transected border (Figure 4c). These data support the results of the immunofluorescence analysis, indicating that ductular reactions only occurred at the transected border.

\section{a-SMA-Positive Hepatic Stellate Cells Were Observed at} the Transected Border

HSCs, which express MMPs ${ }^{26}$ are found in tight association with LPCs. ${ }^{11}$ Therefore, we stained liver sections with an
$\alpha$-SMA antibody, which is a marker of HSC activation, to determine the location of HSCs in the liver. Immunohistochemical staining demonstrated that the number of $\alpha$-SMA-positive cells was significantly increased at the transected border compared with an intact lobe from $48 \mathrm{~h}$ post-procedure (Figures $5 \mathrm{a}$ and $\mathrm{b}$ ). In intact tissue areas, smooth muscle cells of hepatic artery and portal vein branches were mainly $\alpha$-SMA-positive (Supplementary Figure 7). Although infiltrating Kupffer cells also influence the induction of LPCs, there was no significant change in the number of F4/80 positive Kupffer cells in the transected border or intact lobe (Supplementary Figures 8a and b). 
MMP-9 produced by infiltrating Kupffer cells is critical for LPC induction in the chronic liver injury mouse model. ${ }^{27}$ Based on the lack of $\mathrm{F} 4 / 80$ positive cell infiltration, we examined localization of MMP-9 producing cells at the transected border. Immunohistochemical staining revealed that infiltrating cells expressed MMP-9 mainly at the transected border (Figure 5c), which is consistent with the area in which $\alpha$-SMA-positive cells were located (Figure 5a), suggesting that a substantial number of $\alpha$-SMA-positive cells at the transected border may contribute to ECM remodeling.

\section{TNF- $a$ Triggers Proliferation of Mouse LPCs via IKK/NFKB Signaling}

Our experiments suggest that infiltrating cells at the transected border should contribute to ECM remodeling that induces LPCs to participate in ductular reactions. Inflammatory responses associated with ECM remodeling are
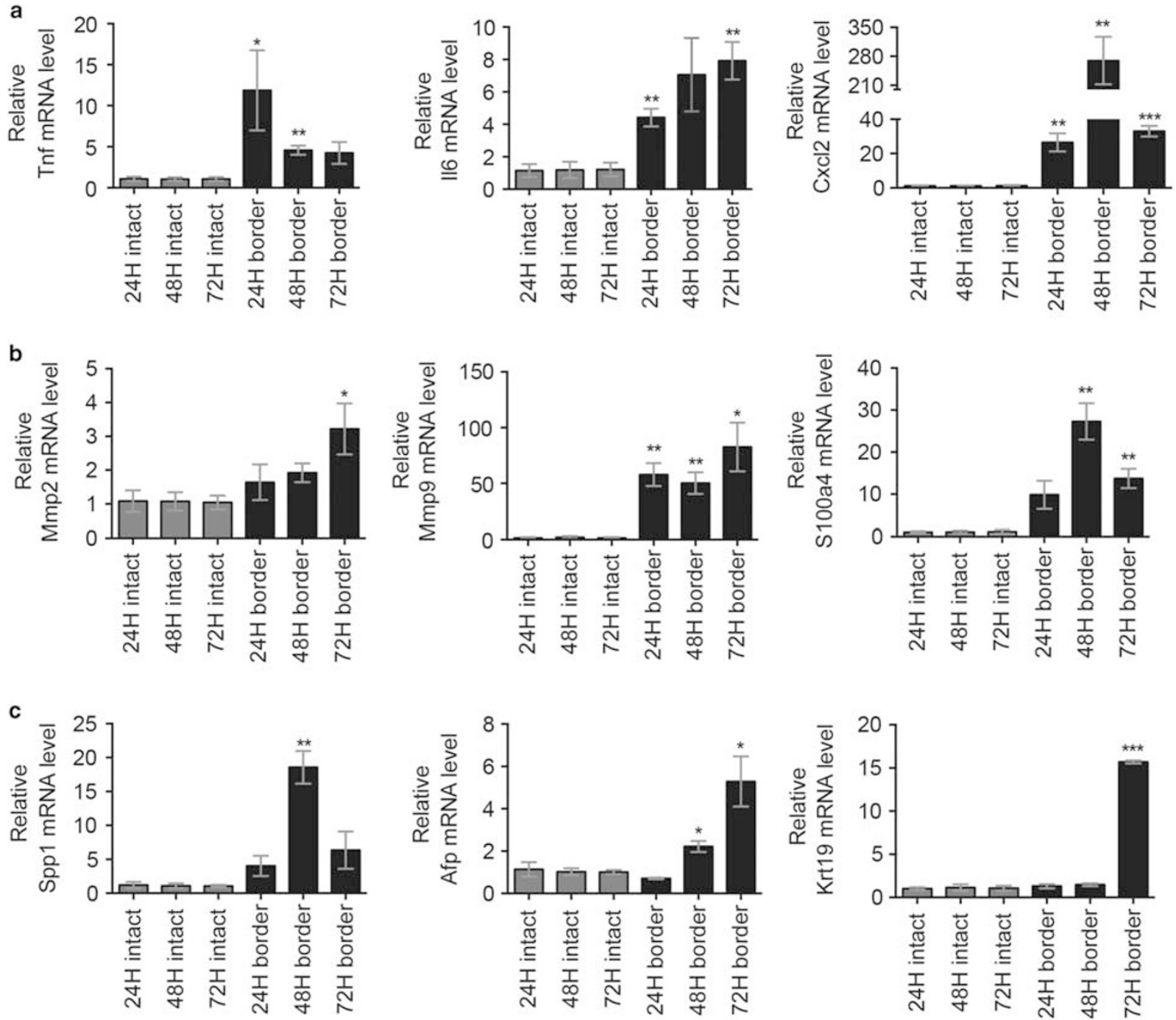

Figure 4 Gene expression analysis of samples taken from the transected border and intact lobe. (a) Expression of Tnf, II6, and Cxcl2 mRNA. (b) Expression of Mmp2, Mmp9, and S100a4 mRNA. (c) Expression of Spp1, Afp, and Krt19 mRNA. Fold induction was estimated for the values of intact lobe at each time point after normalization to $18 \mathrm{~s}$ mRNA levels. Student's $t$-test was performed. Results are represented as mean \pm s.e.m. $(n=3)$, ${ }^{*} P<0.05$, ${ }^{* *} P<0.01,{ }^{* * *} P<0.001$.

Figure 5 Immunohistochemical analysis of $a$-SMA and MMP-9. (a) Histological examination of $a$-SMA-positive area in the transected area at the indicated time points. (b) Quantitative analysis of $a$-SMA-positive area using ImageJ program. Ten randomly selected high-power fields were quantified. Scale bar, $200 \mu \mathrm{m}$. Student's $t$-test was performed. Mean \pm s.e.m., ${ }^{*} P<0.05,{ }^{* *} P<0.01,{ }^{* * *} P<0.001$, n.s., not significant. (c) Histological examination of MMP-9 in the transected area at the indicated time points. Scale bar, $200 \mu \mathrm{m}$. 
a
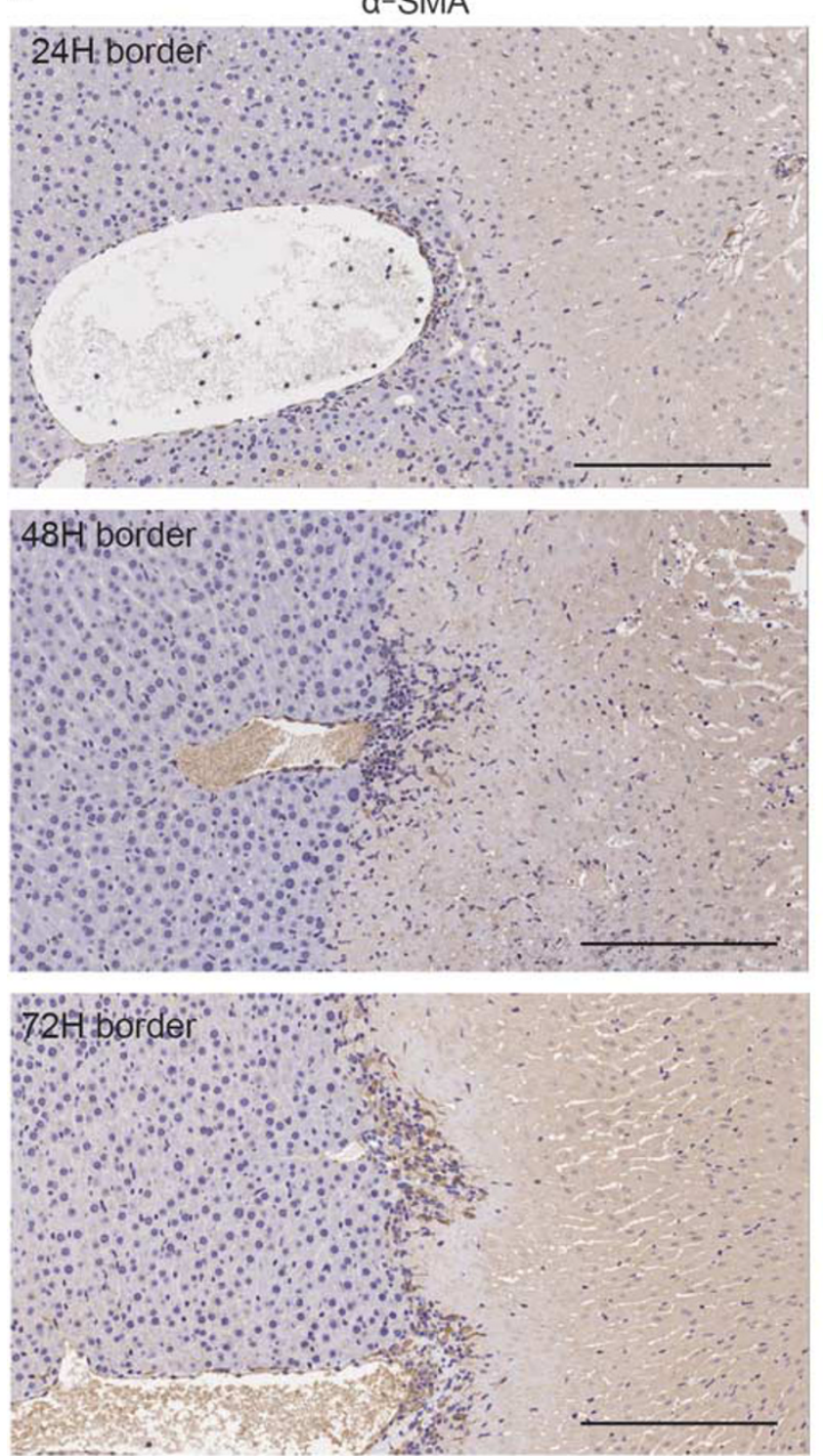

C
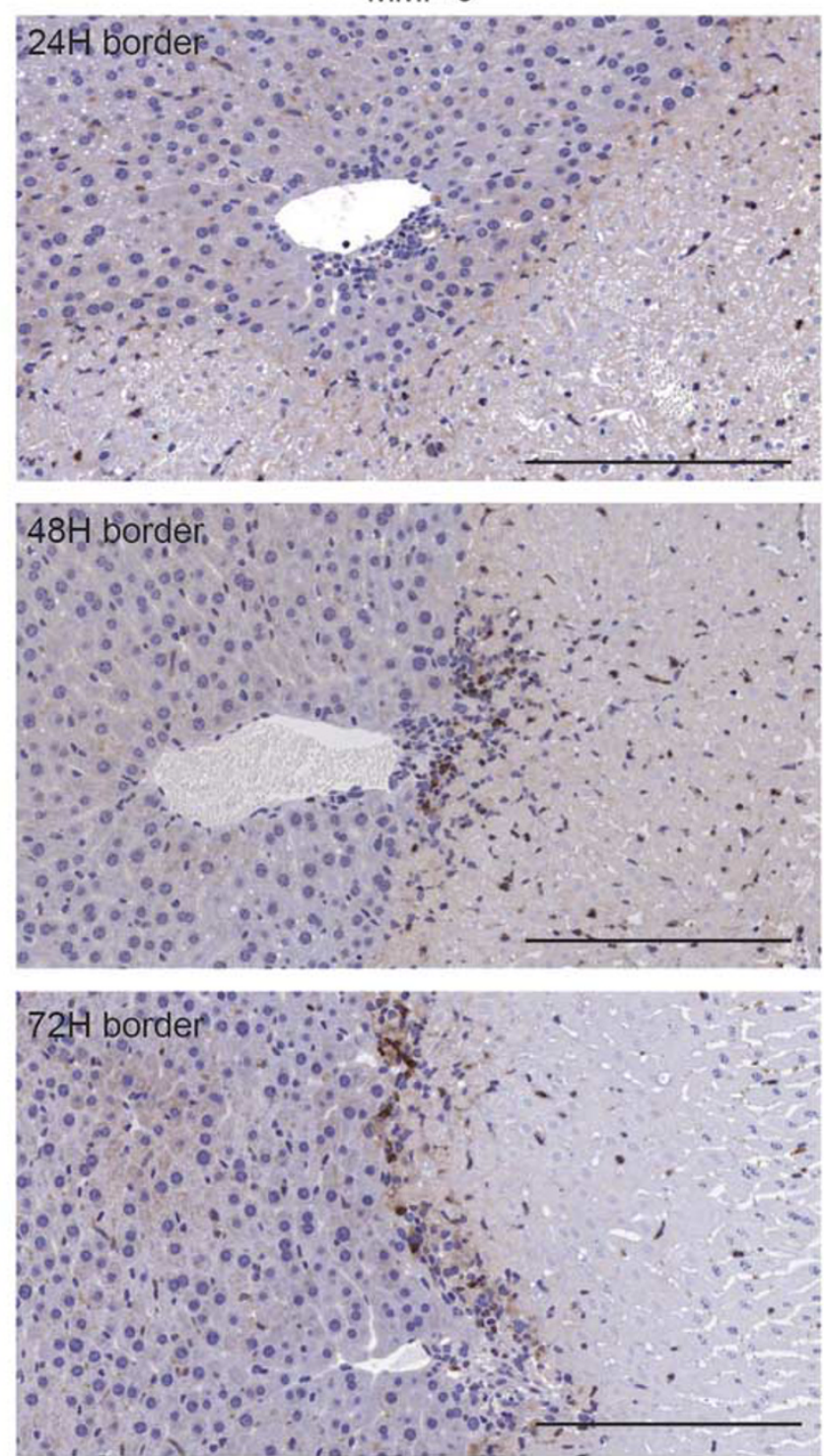

b

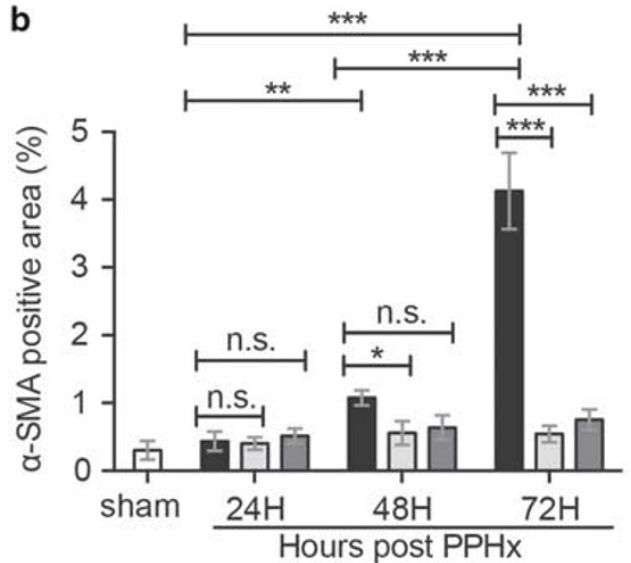

transected border

distant from transected border

intact lobe 

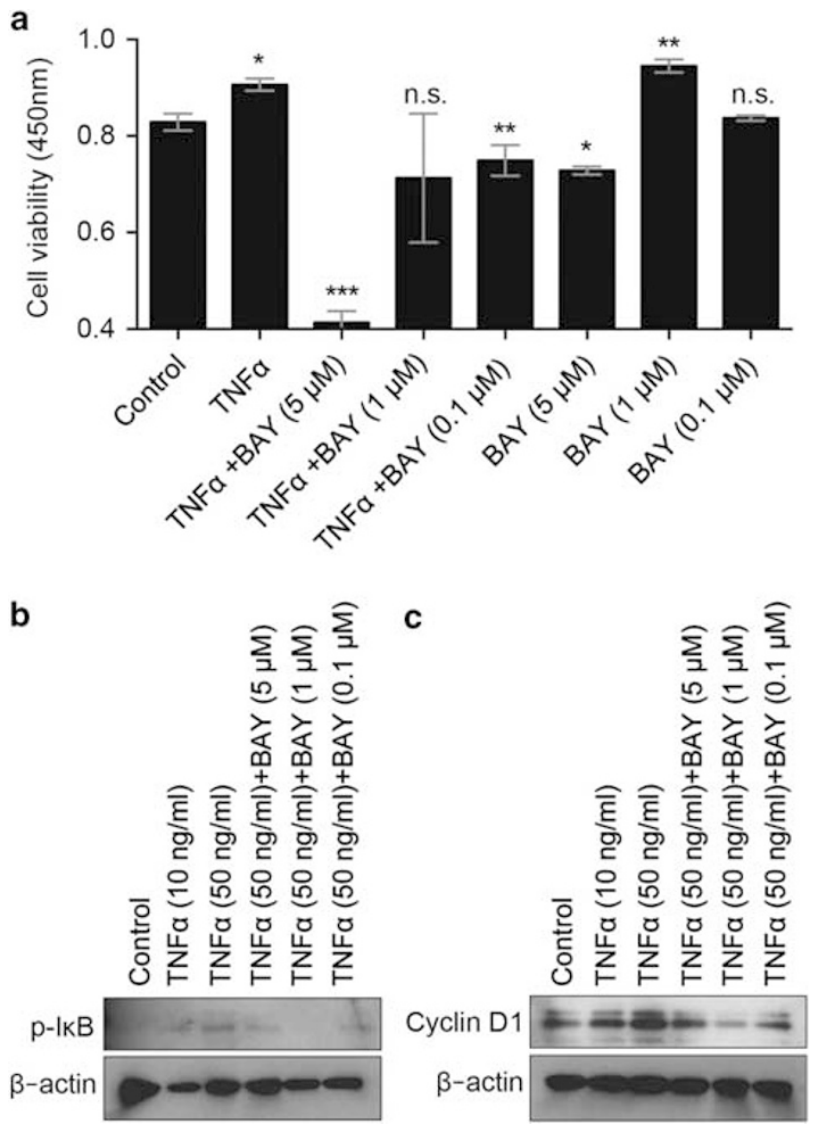

Figure 6 Effects of TNF- $a$ and IKB- $a$ inhibitor BAY11-7082 on mouse LPC proliferation. (a) Cell viability of LPCs was estimated in response to treatment with $50 \mathrm{ng} / \mathrm{ml}$ TNF- $a$, BAY11-7082, and their combination. Student's $t$-test was performed to compare each treated sample to control sample. Results are represented as mean \pm s.e.m. $(n=3),{ }^{*} P<0.05$, ${ }^{* *} P<0.01,{ }^{* * *} P<0.001$. (b) and (c) Western blot analysis was performed using cell lysates of LPCs and antibodies specific for $\mathrm{p}-\mathrm{I}_{\kappa \mathrm{B}}-a$ and Cyclin D1 as indicated. LPCs were treated with $10 \mathrm{ng} / \mathrm{ml}$ or $50 \mathrm{ng} / \mathrm{ml}$ TNF- $a$ with or without $0.1 \mu \mathrm{M}, 1 \mu \mathrm{M}$, or $5 \mu \mathrm{M}$ BAY11-7082. $\beta$-actin was used as the internal control.

considered to be important components in the induction of LPCs. ${ }^{12}$ To determine whether LPC proliferation is triggered by inflammatory cytokines, we assessed if the administration of TNF- $\alpha$ and IL-6 induces LPC proliferation in vitro. Administration of $50 \mathrm{ng} / \mathrm{ml}$ of TNF- $\alpha$ induced LPC proliferation by stimulating the activation of $I \kappa B$ (Figures $6 a$ and $b$ ). In contrast, however, administration of IL-6 did not induce LPC proliferation (data not shown). In a separate study, we found that CXCL2 induced mouse LPC proliferation in vitro, suggesting the involvement of an inflammatory response in LPC-associated liver regeneration (Kakisaka et al., unpublished data).

We next examined whether the proliferative effect of TNF- $\alpha$ was abolished by the NF $\kappa$ B inhibitor BAY11-7082. It has been reported that BAY11-7082 suppresses hepatocyte proliferation at $10 \mu \mathrm{M} .^{28}$ Incubation of LPCs with $5 \mu \mathrm{M}$ of BAY11-7082 for $24 \mathrm{~h}$ induced the suppression of proliferation, whereas lower concentrations (range $0.1-1 \mu \mathrm{M}$ ) exhibited no suppressive effect (Figure 6a). Administration of $50 \mathrm{ng} / \mathrm{ml}$ of TNF- $\alpha$ stimulated the phosphorylation of I $\kappa \mathrm{B}$ $\alpha$ (Figure 6b) and increased Cyclin D1 expression (Figure 6c). Moreover, the induction of these genes at the protein level was suppressed by co-treatment with as low as $0.1 \mu \mathrm{M}$ of BAY11-7082 (Figures 6b and c).

\section{DISCUSSION}

In this report, we evaluated a liver wound healing process after the simultaneous induction of liver volume loss and tissue damage. Using a murine liver injury model that mimics the actual human surgical procedure, we demonstrated that both hypertrophy and proliferation of hepatocytes were induced simultaneously in the entire liver by local severe tissue damage as a part of liver regeneration. At the transected border, ductular reactions were observed at $48-72 \mathrm{~h}$ after the PPHx along the damaged border. Ductular reactions, which contain a population of LPCs, are thought to occur diffusely when the proliferation of hepatocytes is impaired by both acute and chronic liver damage. ${ }^{6,10}$ Our results suggest that ductular reactions could be induced even if the damage is limited to a small area of the liver. Although the differentiation of LPCs into hepatocytes and cholangiocytes has been controversial, ${ }^{9,29,30}$ based on our results, it is conceivable that LPCs participate in the tissue repair process after PPHx.

We have recently shown that a unique stem cell population in adult human bone marrow mesenchymal stem cells, namely multilineage-differentiating stress enduring (Muse) cells, integrate near the transected border and subsequently differentiate into major liver components after PPHx. ${ }^{31}$ In that study, human muse cells were injected into the tail vein of SCID mice $24 \mathrm{~h}$ after PPHx, and we found that they engrafted preferentially into the damaged border and expressed liver progenitor markers $48 \mathrm{~h}$ after transplantation. From these previous observations, we hypothesize that the wound region provides a microenvironment that mobilizes LPCs.

Based on our microarray results, we found that the PPHx induced the expression of inflammatory cytokine genes, including $T n f$, Il6, and $C x c l 2$, at the wound site compared with intact lobes. The involvement of TNF- $\alpha$, IL- 6 , and other inflammatory cytokines has been implicated in LPC proliferation when injury-induced proliferation of hepatocytes is impaired. ${ }^{32-34}$ Our in vitro experiment showed that TNF- $\alpha$ induced LPC proliferation. Indeed, the $\mathrm{NF} \kappa \mathrm{B}$ inhibitor BAY11-7082 inhibited Cyclin D1 induction at a concentration that did not cause cytotoxicity. These results suggest that TNF- $\alpha$ signaling through $\mathrm{NF} \kappa \mathrm{B}$ activation plays a role in the proliferation of LPCs. ${ }^{33} \mathrm{~A}$ recent report demonstrated that TNF- $\alpha$ was responsible for inducing the morphological change of hepatocytes into biliary epithelial cells in vitro. ${ }^{21}$ Another recent study also demonstrated that mature hepatocytes could convert into LPCs to form bile duct-like structures in chronic injury models. ${ }^{35}$ These observations suggest that LPCs induced by pro-inflammatory cytokines are important factors in the generation of ductular reactions. 
We also observed that a significant upregulation of ECMassociated genes, such as Mmp2, Mmp9, and S100a4, occurred simultaneously with the induction of ductular reactions. MMP-2 and MMP-9, which are involved in the degradation of the ECM, are closely associated with LPC mobilization. ${ }^{36}$ In addition, S100a4 promotes liver fibrosis by activating HSCs. ${ }^{22}$ Immunohistochemical staining of MMP-9 revealed that infiltrating cells expressed MMP-9 mainly at the transected border, which was consistent with the area in which both $\alpha$-SMA-positive cells and ductular reactions were located. Our PPHx model induced severe hepatic parenchymal damage at the transected site. Pathological features of the transected site resemble acetaminophen-induced liver injury, ${ }^{37,38}$ whereby $\mathrm{A} 6 / \mathrm{CK}$-positive cells were observed as early as $3 \mathrm{~h} .{ }^{37}$ In the PPHx model, no CK19-positive cells were seen at the transected border up to $24 \mathrm{~h}$. The CK19positive cells were detected between 48 and $72 \mathrm{~h}$ after PPHx with infiltrating inflammatory cells at the transected border. Moreover, those CK19-positive ductular cells also expressed A6. These results are consistent with recent studies showing that ductular reactions appear to be tightly associated with HSCs, macrophages, and the ECM during the response to liver injury. ${ }^{11,39}$ Therefore, although our PPHx is a distinct model of liver injury using this procedure, the pathological features of severe injury by acetaminophen are not entirely dissimilar to those of PPHx. Taken together, we suggest that expansion of ductular reactions in the wound region is regulated by cell-cell and cell-matrix interactions, which are triggered, at least in part, by $\mathrm{TNF} \alpha / \mathrm{NF} \kappa \mathrm{B}$ inflammatory pathway activation.

In conclusion, our PPHx model triggered local ECM remodeling that resulted in ductular reactions. These processes occurred during the tissue repair process in local inflammatory responses as well as compensatory hepatocyte hypertrophy of the entire liver. These findings may provide insight into elucidating the mechanism of hepatic tissue repair and regeneration of the liver after PPHx.

Supplementary Information accompanies the paper on the Laboratory Investigation website (http://www.laboratoryinvestigation.org)

\section{ACKNOWLEDGMENTS}

We thank Dr Valentina Factor for providing the A6 antibody. We also thank Dr Kazuyuki Ishida for advice on pathological findings of the liver sections and Noriyuki Yamada for assistance with preparation of frozen sections. This work was supported by a Grant-in Aid for Scientific Research (C) 25461959 and (C) 25461008, and grant from Keiryokai Research Foundation No. 132.

\section{DISCLOSURE/CONFLICT OF INTEREST}

The authors declare no conflict of interest.

1. Clavien PA, Petrowsky $\mathrm{H}$, DeOliveira $\mathrm{ML}$, et al. Strategies for safer liver surgery and partial liver transplantation. N Engl J Med 2007;356: 1545-1559.

2. Miyaoka $\mathrm{Y}$, Ebato $\mathrm{K}$, Kato $\mathrm{H}$, et al. Hypertrophy and unconventional cell division of hepatocytes underlie liver regeneration. Curr Biol 2012;22: 1166-1175.
3. Mitchell $\mathrm{C}$, Willenbring $\mathrm{H}$. A reproducible and well-tolerated method for 2/3 partial hepatectomy in mice. Nat Protoc 2008;3:1167-1170.

4. Hori T, Ohashi N, Chen F, et al. Simple and sure methodology for massive hepatectomy in the mouse. Ann Gastroenterol 2011;24: 307-318.

5. Malato $\mathrm{Y}$, Naqvi S, Schurmann $\mathrm{N}$, et al. Fate tracing of mature hepatocytes in mouse liver homeostasis and regeneration. J Clin Invest 2011;121:4850-4860.

6. Roskams TA, Libbrecht L, Desmet VJ. Progenitor cells in diseased human liver. Semin Liver Dis 2003;23:385-396.

7. Duncan AW, Dorrell C, Grompe M. Stem cells and liver regeneration. Gastroenterology 2009;137:466-481.

8. Miyajima A, Tanaka M, Itoh T. Stem/progenitor cells in liver development, homeostasis, regeneration, and reprogramming. Cell Stem Cell 2014;14:561-574.

9. Lu WY, Bird TG, Boulter L, et al. Hepatic progenitor cells of biliary origin with liver repopulation capacity. Nat Cell Biol 2015;17:971-983.

10. Gouw AS, Clouston AD, Theise ND. Ductular reactions in human liver: diversity at the interface. Hepatology 2011;54:1853-1863.

11. Lorenzini S, Bird TG, Boulter L, et al. Characterisation of a stereotypical cellular and extracellular adult liver progenitor cell niche in rodents and diseased human liver. Gut 2010;59:645-654.

12. Kim KH, Chen CC, Alpini G, et al. CCN1 induces hepatic ductular reaction through integrin alphavbeta(5)-mediated activation of NF-kappaB. J Clin Invest 2015;125:1886-1900.

13. Okabe $M$, Tsukahara $Y$, Tanaka $M$, et al. Potential hepatic stem cells reside in EpCAM+ cells of normal and injured mouse liver. Development 2009;136:1951-1960.

14. Pierce JW, Schoenleber R, Jesmok G, et al. Novel inhibitors of cytokineinduced IkappaBalpha phosphorylation and endothelial cell adhesion molecule expression show anti-inflammatory effects in vivo. J Biol Chem 1997;272:21096-21103.

15. Ishiyama M, Miyazono $\mathrm{Y}$, Sasamoto $\mathrm{K}$, et al. A highly water-soluble disulfonated tetrazolium salt as a chromogenic indicator for $\mathrm{NADH}$ as well as cell viability. Talanta 1997:44:1299-1305.

16. Takase HM, Itoh $\mathrm{T}$, Ino $\mathrm{S}$, et al. FGF7 is a functional niche signal required for stimulation of adult liver progenitor cells that support liver regeneration. Genes Dev 2013;27:169-181.

17. Inagaki NF, Inagaki FF, Kokudo N, et al. Use of mouse liver mesothelial cells to prevent postoperative adhesion and promote liver regeneration after hepatectomy. J Hepatol 2015;62:1141-1147.

18. Baldin V, Lukas J, Marcote MJ, et al. Cyclin D1 is a nuclear protein required for cell cycle progression in G1. Genes Dev 1993;7: 812-821.

19. Knight B, Matthews VB, Akhurst B, et al. Liver inflammation and cytokine production, but not acute phase protein synthesis, accompany the adult liver progenitor (oval) cell response to chronic liver injury. Immunol Cell Biol 2005;83:364-374.

20. Van Hul NK, Abarca-Quinones J, Sempoux C, et al. Relation between liver progenitor cell expansion and extracellular matrix deposition in a CDE-induced murine model of chronic liver injury. Hepatology 2009;49:1625-1635.

21. Nagahama $Y$, Sone $M$, Chen $X$, et al. Contributions of hepatocytes and bile ductular cells in ductular reactions and remodeling of the biliary system after chronic liver injury. Am J Pathol 2014;184: 3001-3012.

22. Chen L, Li J, Zhang J, et al. S100A4 promotes liver fibrosis via activation of hepatic stellate cells. J Hepatol 2015;62:156-164.

23. Wang $X$, Kiyokawa $H$, Dennewitz $M B$, et al. The Forkhead Box $\mathrm{m} 1 \mathrm{~b}$ transcription factor is essential for hepatocyte DNA replication and mitosis during mouse liver regeneration. Proc Natl Acad Sci USA 2002;99:16881-16886.

24. Li J, Campbell JS, Mitchell C, et al. Relationships between deficits in tissue mass and transcriptional programs after partial hepatectomy in mice. Am J Pathol 2009;175:947-957.

25. Arai M, Yokosuka O, Chiba T, et al. Gene expression profiling reveals the mechanism and pathophysiology of mouse liver regeneration. J Biol Chem 2003;278:29813-29818.

26. Benyon RC, Arthur MJ. Extracellular matrix degradation and the role of hepatic stellate cells. Semin Liver Dis 2001;21:373-384.

27. Ishikawa T, Factor VM, Marquardt JU, et al. Hepatocyte growth factor/c-met signaling is required for stem-cell-mediated liver regeneration in mice. Hepatology 2012;55:1215-1226. 
28. Jimenez-Lopez JM, Wu D, Cederbaum Al. Synergistic toxicity induced by prolonged glutathione depletion and inhibition of nuclear factorkappaB signaling in liver cells. Toxicol In Vitro 2008;22:106-115.

29. Schaub JR, Malato Y, Gormond C, et al. Evidence against a stem cell origin of new hepatocytes in a common mouse model of chronic liver injury. Cell Rep 2014;8:933-939.

30. Yanger K, Knigin D, Zong Y, et al. Adult hepatocytes are generated by self-duplication rather than stem cell differentiation. Cell Stem Cell 2014;15:340-349.

31. Katagiri $\mathrm{H}$, Kushida $\mathrm{Y}$, Nojima $\mathrm{M}$, et al. A distinct subpopulation of bone marrow mesenchymal stem cells, muse cells, directly commit to the replacement of liver components. Am J Transplant 2016;16:468-483.

32. Matthews VB, Klinken E, Yeoh GC. Direct effects of interleukin-6 on liver progenitor oval cells in culture. Wound Repair Regen 2004;12: 650-656.

33. Kirillova I, Chaisson M, Fausto N. Tumor necrosis factor induces DNA replication in hepatic cells through nuclear factor kappaB activation. Cell Growth Differ 1999;10:819-828.
34. Jakubowski A, Ambrose C, Parr $M$, et al. TWEAK induces liver progenitor cell proliferation. J Clin Invest 2005;115:2330-2340.

35. Tarlow BD, Pelz C, Naugler WE, et al. Bipotential adult liver progenitors are derived from chronically injured mature hepatocytes. Cell Stem Cell 2014;15:605-618.

36. Pham Van T, Couchie D, Martin-Garcia N, et al. Expression of matrix metalloproteinase- 2 and -9 and of tissue inhibitor of matrix metalloproteinase-1 in liver regeneration from oval cells in rat. Matrix Biol 2008;27:674-681.

37. Kofman AV, Morgan G, Kirschenbaum A, et al. Dose- and timedependent oval cell reaction in acetaminophen-induced murine liver injury. Hepatology 2005;41:1252-1261.

38. Kuwahara R, Kofman AV, Landis CS, et al. The hepatic stem cell niche: identification by label-retaining cell assay. Hepatology 2008;47: 1994-2002.

39. Kallis YN, Robson AJ, Fallowfield JA, et al. Remodelling of extracellular matrix is a requirement for the hepatic progenitor cell response. Gut 2011;60:525-533. 